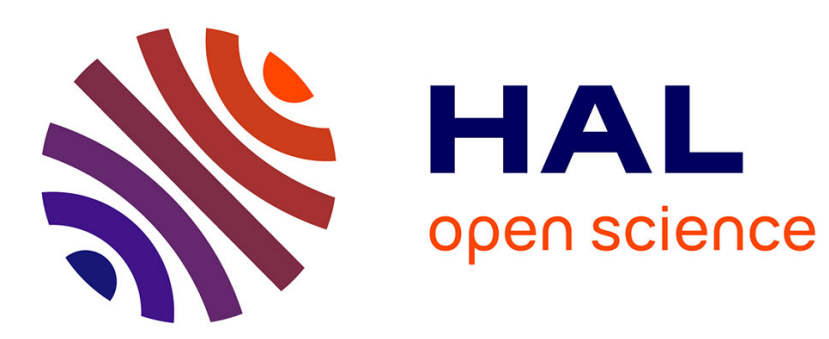

\title{
Onset of Granular Flows on an Inclined Rough Surface: Dilatancy Effects
}

Olivier Pouliquen, Nathalie Renaut

\section{To cite this version:}

Olivier Pouliquen, Nathalie Renaut. Onset of Granular Flows on an Inclined Rough Surface: Dilatancy Effects. Journal de Physique II, 1996, 6 (6), pp.923-935. 10.1051/jp2:1996220 · jpa-00248341

\section{HAL Id: jpa-00248341 https://hal.science/jpa-00248341}

Submitted on 1 Jan 1996

HAL is a multi-disciplinary open access archive for the deposit and dissemination of scientific research documents, whether they are published or not. The documents may come from teaching and research institutions in France or abroad, or from public or private research centers.
L'archive ouverte pluridisciplinaire HAL, est destinée au dépôt et à la diffusion de documents scientifiques de niveau recherche, publiés ou non, émanant des établissements d'enseignement et de recherche français ou étrangers, des laboratoires publics ou privés. 


\title{
Onset of Granular Flows on an Inclined Rough Surface: Dilatancy Effects
}

\author{
Olivier Pouliquen $\left({ }^{*}\right)$ and Nathalie Renaut \\ McGill University, Department of Civil Engineering and Applied Mechanics, Montreal, QC, \\ H3A 2K6, Canada
}

(Received 6 December 1995, received in final form 19 February 1996, accepted 27 February 1996)

PACS.83.70.Fn - Granular solids

PACS.46.10.+z - Mechanics of discrete systems

\begin{abstract}
The onset of the flow of a two-dimensional granular material resting on an inclined rough surface is studied in both experiments and numerical simulations. The critical angle $\theta_{c}$ at which the particles start to flow is found to increase when the initial thickness of the layer decreases, showing that the strength of the material, i.e. the internal friction angle, is greater near the rough surface than in the bulk. This phenomenon is shown to be connected to the dilatancy occurring in the medium when it starts to flow: in a thin layer of material the rigidity of the surface prevents local rearrangements of the particles and thus imposes a greater level of dilatancy, leading to a larger effective friction angle. This robust effect is also observed with three-dimensional granular materials involving irregular shaped particles.
\end{abstract}

\section{Introduction}

The understanding of the behavior of granular materials is of prime importance for many industrial processes (storage of seeds, handling of pharmaceutical powders, . ) as well as in the description and forecast of geophysical events (landslides, snow avalanches, ...). However, our knowledge about the dynamics of particulate systems is still very poor compared to other media. One major difficulty is that they belong to an intermediate state of matter [1] since they can flow like a fluid but they can also sustain internal stresses like a solid. An important problem is to predict the transition between solid-like and fluid-like behavior. The avalanches occurring at the free surface of a sand heap when the slope is greater than a critical value, is one of the most studied example of such a transition [2-5].

The ability of a granular material to sustain internal shear stresses up to a certain level is generally described using a Coulomb criterion. The latter stipulates that yielding will occur along a certain plane if the shear stress $\tau$ reaches a value corresponding to the product of the normal stress $\sigma$ with the tangent of the internal static friction angle $\phi$ such that

$$
\tau=\sigma \tan \phi \text {. }
$$

The angle $\phi$ is a macroscopic parameter that provides a measure of the strength of the material. From the microscopic point of view, this strength can be attributed to two factors: the friction

$\left({ }^{*}\right)$ Permanent address: LaDHyX, École Polytechnique, 91128 Palaiseau Cedex, France Author for correspondence (e-mail: olivier@ladhyx.polytechnique.fr) 
between individual grains and the geometrical interlocking between the particles. The former is solely a property of the material whereas the latter is purely geometric and is known as the Reynolds dilatancy. Reynolds [6] was the first to observe that in order to change its shape a granular material has to dilate. In a dense packing the particles are trapped between their neighbors and in order to move they have to get one over the other, leading to an increase of the volume occupied by the particles. Due to this geometrical constraint [7], a pile of frictionless grains will have a non zero macroscopic friction angle. The strong influence of the dilatancy on the behavior of granular materials has led to many studies concerning its estimation for packing of disks or spheres [7-10] and concerning constitutive equations trying to take it into account [11].

The macroscopic friction angle $\phi$, which takes into account both the friction between particles and the dilatancy, describes the strength of the material in the bulk. However, in many problems such as "inclined chute flows" [12-15] one has to take into account the interaction between the grains and a surface. In the continuum mechanics framework this interaction is usually modelled by the mean of another friction angle $\delta$ called the "bed friction angle". The usual way to measure $\delta$ consists in inclining the bed until the granular material starts to slide on the surface. The angle corresponding to this event is the bed friction angle. For inclined chute flows involving a smooth bed the description in term of a bed friction angle seems to be appropriate.

We present in this paper both experiments and numerical simulations of the onset of the flow on an inclined rough surface. The system consist in inclining a rough surface covered by a layer of granular material and measuring the critical angle at which it starts to flow. The role of the bed roughness has been previously studied for a single particle flowing down a plane [16], but to our knowledge has never been investigated in the case of a layer of granular material. We show in this paper that the bed roughness dramatically affects the behavior of the material at the onset of the flow: the critical angle at which the granular flow is initiated does not have a unique value as in the case of a smooth bed, but it varies with the initial thickness of the piling. The thinner the initial layer of material, the greater the bed inclination necessary to initiate flow. A description in term of a bed friction angle is thus no longer valid. However using molecular-dynamic simulations we were able to interpret these results as a variation of the internal friction angle $\phi$ arising from a variation of the dilatancy of the material close to the bed.

The paper is organized as follows. In the second Section, two-dimensional experiments carried out with cylinders are described. The critical angle at which the cylinders start to flow is found to strongly depend on the thickness of the pile. Details of the numerical simulations and of their results are given in Section 3. In Section 4, calculations of the dilatancy are performed and we discuss its role in the increase of the friction angle close to the bed. Threedimensional experiments using glass beads and crushed walnut shells are presented in Section 5. Final remarks and conclusion are given in Section 6 .

\section{Two-Dimensional Experiments}

The two-dimensional experiments were carried out with $6 \mathrm{~cm}$ long aluminum cylinders. In order to avoid the formation of a regular packing the medium consisted of an equal mixture of $2 \mathrm{~mm}$ and $3 \mathrm{~mm}$ diameter cylinders (the mean particle diameter $d$ is $2.5 \mathrm{~mm}$ ). The rods have a density of $2.7 \mathrm{~g} / \mathrm{cm}^{3}$ and the value of the aluminum-aluminum friction angle is $19.5^{\circ}$. The stability of a material layer formed by these rods was studied by the mean of the experimental set-up shown in Figure 1. The bed had a length of $1.2 \mathrm{~m}$ and a width of $8 \mathrm{~cm}$. The cylinders being quite long compare to the depth of the piling, no lateral motion took place and consequently no 


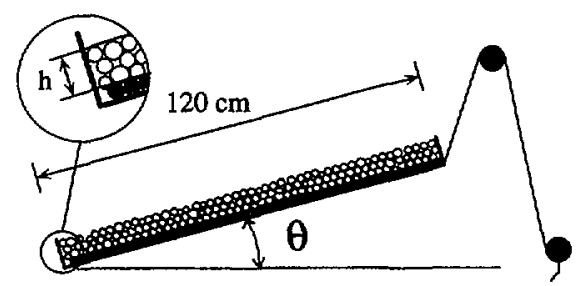

Fig. 1. - Experimental set-up.

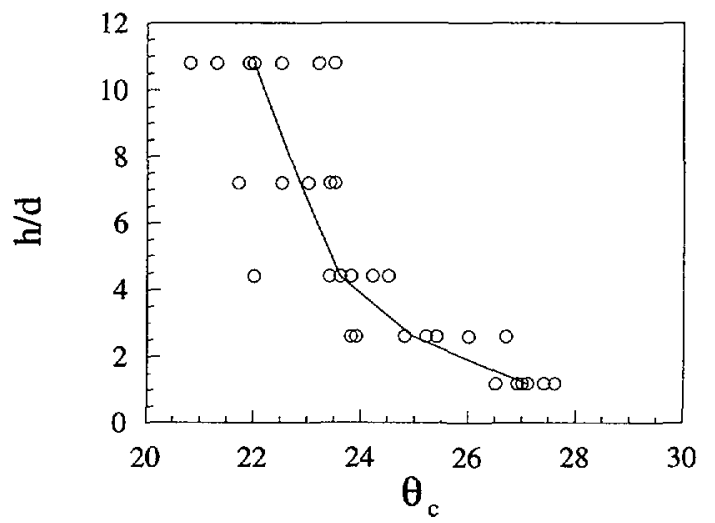

Fig. 2. - Variation of the critical angle $\theta_{c}$ at which the flow starts with the nondimensionalized depth $h / d$ of the material. Data from the two-dimensional experiments.

side-walls were required to hold the rods. The bed surface was roughened by gluing side by side $2.5 \mathrm{~mm}$ diameter plastic cylinders. The plastic-aluminum friction angle happens to be equal to the aluminum-aluminum friction angle i.e. $19.5^{\circ}$. Therefore the use of plastic cylinders at the wall did not cause any problem.

Before each experiment the bed was set to the horizontal and the initially well mixed medium was arranged on the surface such that the depth $h$ was constant over the whole bed. The bed was then raised slowly and carefully by the mean of a wire passing through a pulley (Fig. 1). As the bed was inclined, small events occurred which correspond to local rearrangements of the cylinders. Such events never involved more than 20 particles and led to small displacements of the order of one particle diameter. These motions enable the cylinders to find a new equilibrium position. However, at a specific angle $\theta_{c}$ a big avalanche occurred where more than half of the cylinders flew down the bed. This event was referred to as the onset of the flow $\left({ }^{1}\right)$. At $\theta_{c}$ one clearly observes that the motion is initiated at one point between the two retaining walls that varies from one run to another. No spurious effect due to the retaining wall was observed.

Experiments were performed for different values of the thickness of the pile, $h$, defined as shown in Figure 1. Experiments were performed for $h$ equal to $2.5,6.5,11,18$ and $27 \mathrm{~mm}$ or, in terms of the mean particle diameter, $h / d=1,2.6,4.4,7.2$ and 10.8. Experiments for each case were performed 8 times. The results are presented in Figure 2 . The $x$-axis represents the critical angle $\theta_{c}$ of the onset of the flow, and the $y$-axis is the dimensionless thickness $h / d$. For

$\left({ }^{1}\right)$ Very seldom an event was observed which was too large to be considered as a local rearrangement but too small to be considered as the onset of the flow. In this case, the experiment was stopped and no measurement was recorded. 
a specific depth $h / d$ the range of values of the recorded angles spreads over $3^{\circ}$ or so. This scatter is certainly related to the initial random packing. Some pile are less stable than others and thus start to flow at a lower angle. However, despite this scatter, one clearly observes in Figure 2 that $\theta_{\mathrm{c}}$ increases with the reduction in depth of the granular material. The continuous line represents the mean critical angle which increases from $22^{\circ}$ for $h / d=10.8$ up to $27^{\circ}$ for one layer of particles. In the experiments where the medium has a thickness greater than 4 particle diameters, one observes that some layers of particles remain in equilibrium on the bed even after the occurrence of the big avalanche. This shows that the failure takes place inside the material and not at the interface between the granular medium and the bed.

These observations contrast with the usual continuum description of the effect of a rough surface in terms of a bed friction angle $\delta$. According to such a model, the granular medium should start to flow at an inclination corresponding to $\delta$ independently of the initial thickness of the material. Moreover, the failure should occur at the bed and not inside the medium. This last point suggests that the critical angle $\theta_{c}$ in our experiments measures the internal strength of the material, and thus corresponds to an internal friction angle $\phi$ rather than to a bed friction angle. Thus, Figure 2 can be interpreted as an increase of the internal static friction angle of the medium close to the rough surface.

One have to keep in mind that the notion of friction angle is only valid in the limit of continuum media. In our problem we are dealing with piles less than 10 particles diameters thick, and thus the discrete nature of the material plays a major role. However, although the notion of bed friction angle seems to be no longer valid, the experiments clearly show that a thin layer of materials is able to sustain higher shear stresses than thick ones, suggesting that the notion of internal friction angle is still relevant.

\section{Numerical Simulations}

The avalanche that occurs at the critical inclination angle is a rapid event. It takes place over a short period of time making impossible a precise experimental study of the motion of the particles. In order to carefully study the initiation of the flow we have performed molecular dynamic simulations [17-19]. The code we used is a modified version of the one developed in [20-22]. The simulations were carried out in a two-dimensional configuration and disks were used to represent the granular medium. The computational box consisted of a rough bed made up of 10 particles having a uniform diameter of $0.7 D$, covered by a pile of particles having a uniform random diameter distribution ranging from $0.6 D$ to $0.8 D$, where $D$ is a representative scale of the disk size. Periodic boundary conditions are applied in the eventual direction of the flow. The particles are submitted to an external force field which is gravity and they are also subjected to normal and tangential contact forces that arise from inter-particle interactions. A 'soft' particle approach is used such that multiple contacts of a finite duration take place.

3.1. Contact Force Model. - The problem we are interested in deals with the stability of a layer of material and with the transition between static equilibrium and flow of the medium and thus, special attention has to be given to the contact force model. It must be able to reproduce the static stress distribution without any spurious oscillations which would artificially destabilize the system. Both the normal and tangential forces were thus computed according to the models proposed by Walton and Braun [23]. In their model the normal contact interactions are approximated by a linear spring acting between two rigid bodies such that the normal forces are related to a spring constant $k_{1}$ for the loading phase and a higher stiffness $k_{2}$ during unloading. By using two different spring constants $k_{1}$ and $k_{2}$, for the compression and recovery 
processes, the energy dissipation is incorporate into the collision. The corresponding coefficient of restitution $e$ is then $\sqrt{k_{1} / k_{2}}$.

Tangential forces are also considered to act at the interface between particles and are evaluated using an incrementally slipping model. This model takes account of the elastic deformation that can occur in the tangential direction of the contacts. The tangential force $T$ is evaluated using an effective tangential stiffness $k_{t}$ associated with a non-linear spring. It is incremented at each time step as follows:

$$
T_{t+1}=T_{t}+k_{t} \Delta s
$$

where $\Delta s$ is the relative tangential displacement between two time step. The effective stiffness $k_{t}$ has an initial value of $k_{o}$ and decreases with tangential displacement until it becomes effectively zero at full sliding, when the tangential force $T$ is equal to the normal force $N$ times the coefficient of friction $\mu: T=\mu N$. The effective stiffness is given by:

$$
k_{t}= \begin{cases}k_{0}\left(1-\frac{T-T^{\star}}{\mu N-T^{*}}\right)^{\gamma} & \text { for } T \text { increasing } \\ k_{0}\left(1-\frac{T^{*}-T}{\mu N+T^{*}}\right)^{\gamma} & \text { for } T \text { decreasing },\end{cases}
$$

The variable $T^{*}$ is initially zero and takes the value of $T$ whenever the slip reverses direction. As in [23] we used the value $\gamma=1$ for the calculations. Whenever there is a change in the normal force, $T$ and $T^{\times}$are rescaled proportionally to the normal force.

In the simulations presented here, the parameter $\mu$ was taken to be 0.355 corresponding to the experimental measurement of the aluminum-aluminum friction angle. The coefficient of restitution $e$ was set to 0.5 . Lengths were nondimensionalized by the representative cylinders diameter $D$, and the time by $\sqrt{M / k_{1}}$, where $M$ is the mass of a cylinder of diameter $D$. A value of $2 \times 10^{-4}$ was used for the nondimensionalized gravitational acceleration $g^{*}=M g / D k_{1}$. For the aluminum cylinders used in the experiments, this value of $g^{*}$ corresponds to a normal loading stiffness $k_{1}$ of $22.7 \mathrm{kN} / \mathrm{m}$. This value is certainly less than the real one but it has been found that the various results that can be determined through numerical simulations are not too sensitive to the variations of $k_{1}$ as long as it remains within a certain range where it is sufficiently stiff. Besides, a lower $k_{1}$ permits the use of a larger time step since the contact time for a collision is inversely proportional to $\sqrt{k_{1}}$. For the tangential stiffness we used $k_{0}=0.8 k_{1}$.

3.2. Simulation Procedure And Results. - The particles are initially arranged in a regular array free of contacts and they are given random velocity vectors. Under the action of gravity, they fall on the bed already inclined at $10^{\circ}$ from the horizontal and then they flow downward for a short period of time until a quite stable random arrangement is attained. After the particles have come to rest the bed angle is incremented at a constant rate of $5.9^{\circ} / \mathrm{s}$. The simulations were visualized by means of an animation software, a useful tool for observing the details of the deformation occurring in the medium.

As the bed is raised there is no movement of the particles except for local rearrangements similar to those observed during the experiments, until almost all the particles start to flow. The bed angles corresponding to the beginning of the flow were determined from the visualizations with a precision of $1^{\circ}$. The simulations were done for $1,3,5$ and 8 layers of particles and each case was repeated for several samples.

The critical angles recorded from the simulations for the different initial depths are plotted in Figure 3 where the solid line represents the mean values. One can observe that the scatter of the angles determined from the numerical simulations is larger than in the experimental 


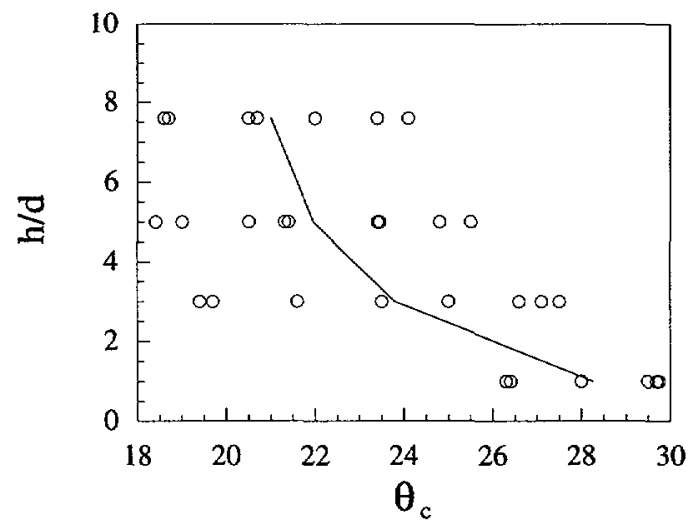

Fig. 3. - Variation of the critical angle $\theta_{c}$ with the nondimensionalized depth $h / d$ of the material. Data from the two-dimensional numerical simulations.

measurements (Fig. 2). This can be explained by the fact that a relatively small number of particles was used in the simulations, such that when an unstable or a very stable region developed it had a significant influence on the whole system because of the periodic boundary conditions. However, as illustrated by the continuous curve in Figure 3, the numerical simulations properly reproduced the experimental behavior, namely the decrease of the effective friction angle as the distance from the bed increases.

\section{Discussion}

4.1. Influence of the Order Induced by the Regular Bed. - Both the numerical simulations and the experiments presented in the previous sections were performed with a regular bumpy bed made of monosized particles perfectly aligned. This type of bed induces some order inside the medium [24] which is lost after few layers of particles due to their random size distribution. One thus observes a variation of the arrangement of the particle across the material from a quasi triangular packing near the bottom surface to a random one in the bulk. Based on these observations we were tempted to explain the reduction of the effective friction angle with distance from the bed as being related to the change in the stacking structure.

In order to check this idea, we have performed some simulations involving irregular bottoms, created by vertically shifting the particles of a regular bottom by a random distance between 0 and 1 particle diameter. The bed generated by this method exhibits the same kind of corrugation found in a layer of particles in the bulk. Simulations were conducted which consisted in inclining a pile of 1,3 and 8 layers of particles until it started to flow. Each case was tested for five samples of particles having a different random bed's structure. The mean critical angle at which the flow was initiated was found to be respectively $31^{\circ}, 26.3^{\circ}$ and $20.4^{\circ}$ for piles made of 1,3 and 8 layers of particles. These simulations show that a similar increase of the effective friction angle is observed with irregular beds as with regular ones. This clearly shows that the geometric structure of the bottom surface is not crucial for our problem.

4.2. Role of the Dilatancy. - We thus performed measurements of the dilatation occurring in the material when it starts to flows, in order to better understand the role of the geometrical interlocking of the particles [6] in our problem. 


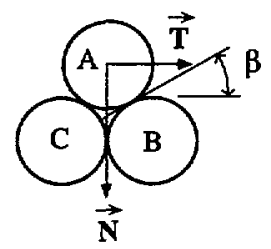

Fig. 4. - Role of the dilatancy.

A simple illustration of how the geometrical interlocking influences the strength of the material has been given by Rowe [8] and is represented in Figure 4. In order to move particle A has to overcome particle $B$. If no rotation of the particles is allowed this motion is possible only if the tangential force $T$ applied to the particle reaches the following critical value:

$$
T=N \tan (\varphi+\beta)
$$

where $N$ is the normal force applied on the particle, $\varphi$ is the particle-particle friction angle, and $\beta$ the angle between the tangential plane between particles $\mathrm{A}$ and $\mathrm{B}$ and the horizontal. This simple model demonstrates how the effective friction coefficient, i.e. the ratio $T / N$, depends on both the friction $(\varphi)$ and the geometry $(\beta)$. In the case of real packing the angle $\beta$ varies from one contact to another. However, when a simple shear strain $\gamma$ is imposed to the pile a coefficient of dilatancy $s$ can be defined in order to quantify the geometrical effect:

$$
s=\frac{\mathrm{d} \epsilon_{\mathrm{v}}}{\mathrm{d} \gamma}
$$

where $\epsilon_{\mathrm{v}}=\ln \left(V / V_{0}\right), V$ and $V_{0}$ being respectively the volume and the initial volume of the sample [10]. The coefficient of dilatancy $s$ can be interpreted as an equivalent of the parameter $\beta$ in the Rowe's Model. Its value is strongly related to the yielding properties of the material under a simple shear [7].

In our problem of the onset of the inclined chute flow, the definition (1) is no longer valid, as the shear strain $\gamma$ is not imposed. It is in fact an unknown quantity which furthermore varies across the pile. In order to quantify the geometrical interlocking effects in our problem we thus proposed the following definition for the coefficient of dilatancy:

$$
s=2 \frac{\mathrm{d} \Delta Y}{\mathrm{~d} \Delta X}
$$

where $\Delta X(t)$ (resp. $\Delta Y(t)$ ) represents the mean displacement parallel (resp. perpendicular) to the bed of the particles present at the free surface. A relation between the two definitions (1) and (2) can be derived if one naively assumed that the deformation profile is as sketched in Figure 5. In such a configuration the volume dilatation rate $\epsilon_{\mathrm{v}}$ calculated in the shear region of depth $D_{\mathrm{f}}$ is equal to $2 \Delta Y(t) / D_{\mathrm{f}}$ (assuming the dilatation to be isotropic and the change in volume to be small) and the shear strain $\gamma$ inside the zone of failure corresponds to $\gamma(t)=\Delta X(t) / D_{\mathrm{f}}$. One then obtains:

$$
\frac{\mathrm{d} \epsilon_{\mathrm{v}}}{\mathrm{d} \gamma}=2 \frac{\mathrm{d} \Delta Y}{\mathrm{~d} \Delta X}
$$

In the limit of a continuum medium the two definitions (1) and (2) are thus equivalent. 


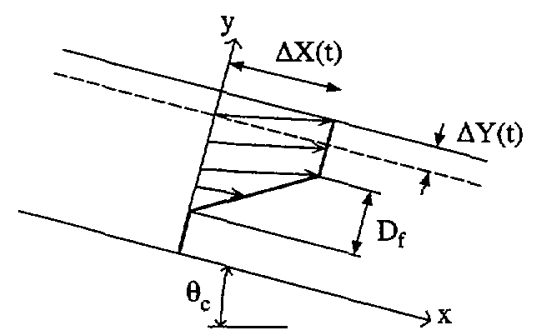

Fig. 5. - Sketch of the initiation of the flow. $\Delta X(t)$ and $\Delta Y(t)$ are the horizontal and vertical displacement of the free surface. $D_{\mathrm{f}}$ is the thickness of the failure zone.

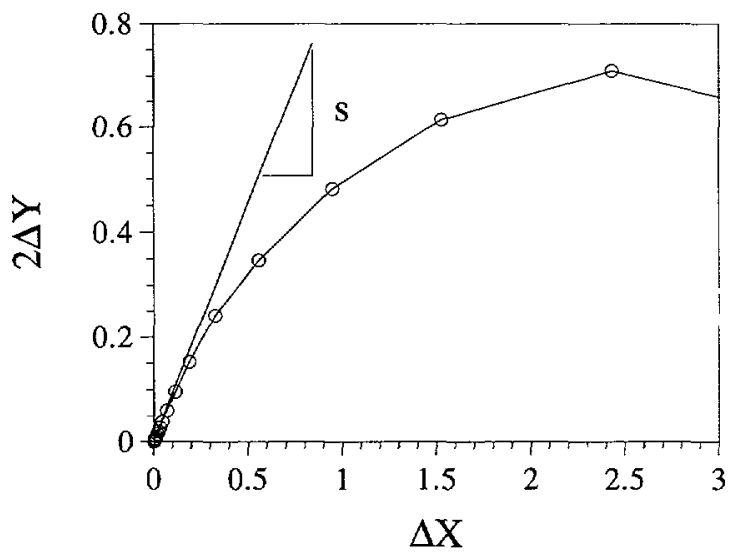

Fig. 6. - Twice the vertical displacement of the free surface $2 \Delta Y(t)$ versus the horizontal displacement $\Delta X(t)$ : the slope at the origin gives the coefficient of dilatancy $s$. Curve obtained from a simulation computed for $h / d=5$.

Figure 6 shows the variation of twice the vertical displacement of the free surface $2 \Delta Y$ versus the horizontal displacement $\Delta X$ obtained from the numerical simulations for a layer having a thickness of 5 particle diameters. Before the flow is initiated the pile is static and the corresponding point in Figure 6 is at the origin $(\Delta X=\Delta Y=0)$. When the critical angle is reached the particles start to flow down the bed, $\Delta X$ increases, and at the same time dilatation occurs yielding an increase in $\Delta Y$. The dilatancy $s$ is obtained by measuring the slope at the origin.

We measured $s$ in all the numerical simulations presented in Section 3 where the thickness of the initial piling varied. The results are summarized in Figure 7 where the horizontal axis is the coefficient $s$ and the vertical axis the thickness of the granular medium in terms of particles diameter. Each point corresponds to one simulation and the continuous line joins the mean dilatancy coefficients for the various thicknesses. From this figure we observe that $s$ exhibits a variation similar to the one observed for the friction angle in Figure 3 . The thinner the pile, the larger dilatancy. This result clearly demonstrates that the critical angle $\theta_{\mathrm{c}}$ at which the pile start to flow is strongly related to the dilatancy $s$ occurring in the pile. This correlation is even more obvious in Figure 8 where the coefficient $s$ is plotted versus the critical angle $\theta_{c}$ for all the simulations. All the points collect on a single curve and we notice that the scatter present in Figures 3 and 6 has disappeared in Figure 8. This shows that there exists a unique 


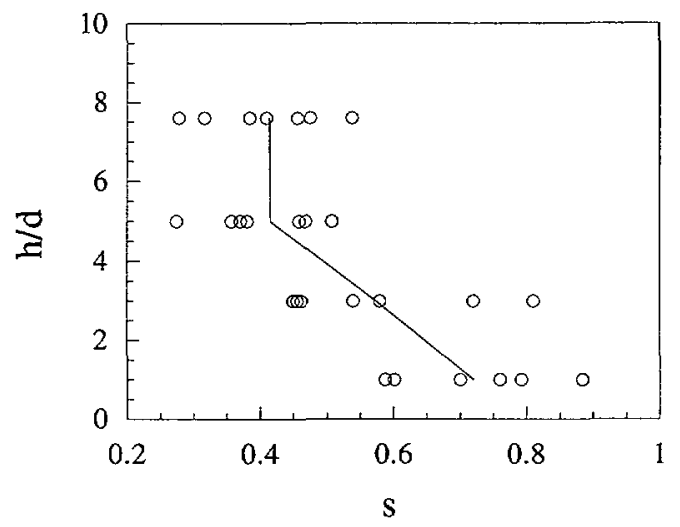

Fig. 7. - Variation of the coefficient of dilatancy $s$ with the thickness of the pile $h / d$. Data from the numerical simulations.

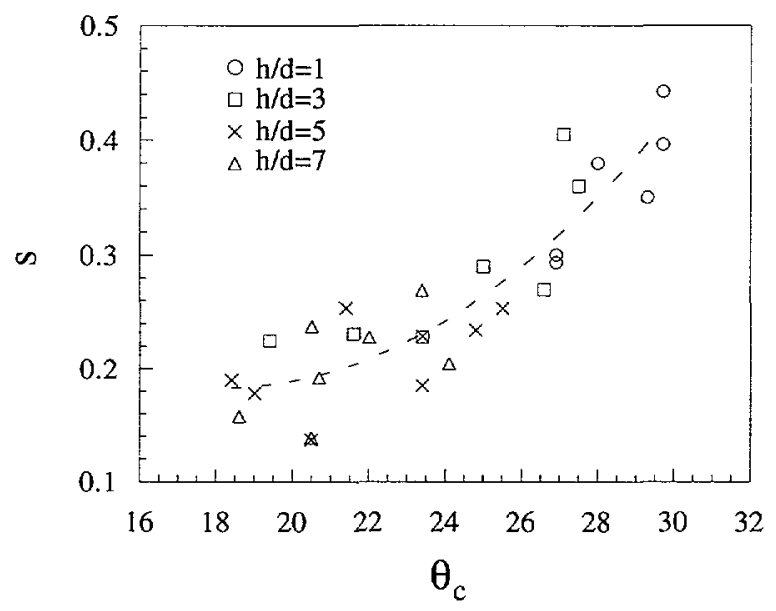

Fig. 8. - The measured coefficient of dilatancy $s$ versus the critical angle $\theta_{c}$ for different thicknesses of the pile. Data from the numerical simulations.

relation between $s$ and $\theta_{c}$. The initiation of the flow on the rough bed is thus driven by the capacity of the material to dilate. The thinner the layer of material, the more it has to dilate in order to shear, the higher the bed inclination necessary to initiate the flow.

The fact that the dilatancy depends on the thickness of the pile can be explained in the following manner. Let us consider a monolayer of particle resting on the bumpy bottom surface. the only way for the particles to move is to overcome the bed particles. The dilatation occurring at the onset of the flow thus corresponds to the jump over the bumpiness of the bed. In the case of a thick pile, the initiation of the flow correspond to the formation of a failure zone inside the material which spreads over several layers of particles $\left({ }^{2}\right)$. In this shear zone, local rearrangements of the particles are possible, which decrease the dilatation occurring

$\left({ }^{2}\right)$ The thickness of the failure has been the subject of many studies in tri-axial configurations [25,26]. The shear band is found to be between 8 and 15 particles diameter thick. 
during the shear. Thus a pile of 4 layers of particles exhibits a dilatation less than 4 times the dilatation observed when one layer starts to flow, which means a lower coefficient of dilatancy. The variation of the material strength close to the bottom surface is thus the result of the rigidity of the surface which prevents local rearrangements of the particles, leading to a higher coefficient of dilatancy, and thus a higher friction angle.

\section{Extensions to 3D Configurations and Dynamic Friction Angles}

The experiments and the numerical simulations presented in Sections 2 and 3 were twodimensional. In this section we present three-dimensional experiments performed with two kinds of material: spherical glass beads having a diameter of $0.45 \mathrm{~mm}$ and crushed walnuts shells which are very irregular shaped particles having a mean diameter of $1.25 \mathrm{~mm}$. The experimental setup consisted of a channel $80 \mathrm{~cm}$ in length and $4 \mathrm{~cm}$ in width with glass sidewalls. The bed was roughened by gluing some of the bulk particles on it. We have performed two kinds of experiments in this channel: the first one is the same as the one presented previously and consists of slowly inclining the channel filed with a pile of constant thickness until flow is initiated. The critical angle is a measure of the effective static friction angle. In the second set of experiments we proceeded in the opposite way. We started with the channel already inclined at a large angle and we created a steady flow by the mean of a bin placed at the top of the channel and filed with the material. The thickness of the flow was controlled by the size of the bin outlet. We then decreased the inclination at a very slow rate such that the flow remained in a quasi-steady regime. The mean velocity of the flow is found to decrease continuously when decreasing the inclination, up to a critical angle at which the flow stops. This angle corresponds to the minimum of the dynamic friction angle [5,27]. We have measured this critical angle for different depths of the flow. The results are summarized in Figures $9 a$ and $b$ for both the glass beads and the walnuts shells. These plots represent the variation of both static (circles) and dynamic (triangles) friction angle with the depth of the flow.

These results first show that the increase of the static friction angle close to the bed which was obtained with disks, is also observed in the three-dimensional systems. For both the glass beads and the walnuts shells, a variation of the critical angle at which the medium starts to flow is observed in the first eight layers of particles. Moreover, the fact that the phenomenon is also observed with the crushed walnuts shells shows that the influence of the roughness of the bed on the strength of the medium is a robust effect independent of the particle shape. Note that the data are less scattered in Figures 9 for $3 \mathrm{D}$ piling than in Figure 2 for the disks. This is probably due to the fact that in a three-dimensional packing there is much smaller probability of peculiar situations such as the formation of arches or holes that can dramatically change the stability properties of the pile. We have not performed dilatancy measurements in those experiments, but one can be easily convinced that the interpretation given in Section 4 is still valid with three-dimensional piling.

A more interesting result coming from this set of experiments is that the dynamic friction angle exhibits the same variation as the static one, i.e. it increases with the decrease in depth of the flow as shown by the triangles in Figures 9. A thin flow of granular material stops at a greater angle than a thick flow. Again this effect is independent of the particle shape. This last observation has strong consequences for the problem of inclined chute flows. First, it implies that the range of bed inclination for which a flow is possible depends on the thickness of the flow as already observed by Vallance [15]. Furthermore, the increase of the dynamic friction angle near the bed certainly affects the whole dynamics of the flow as shown by the following example: let us consider a 10 diameters thick flow of glass beads on a $25^{\circ}$ inclined plane. According to Figure $9 \mathrm{a}$ the flow is possible because the intersection between the lines $h / d=10$ 


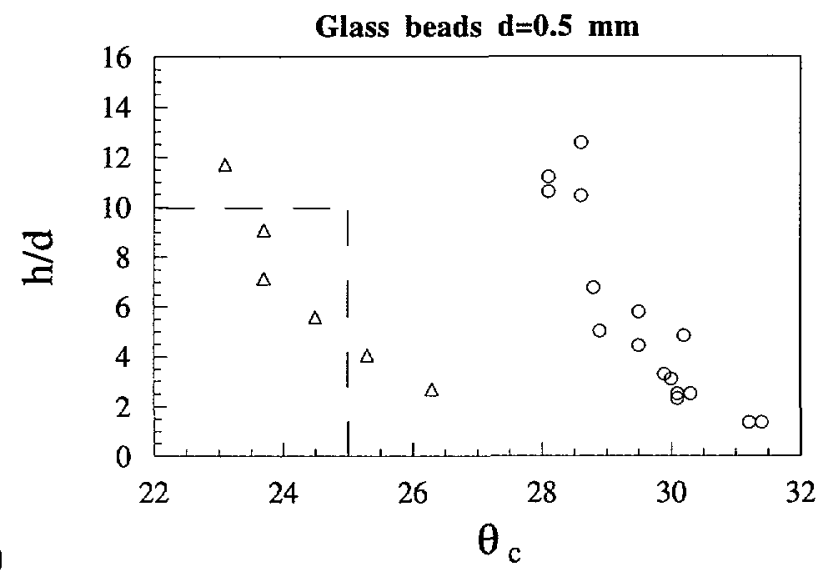

a)

Walnuts shells $\mathrm{d}=1.25 \mathrm{~mm}$

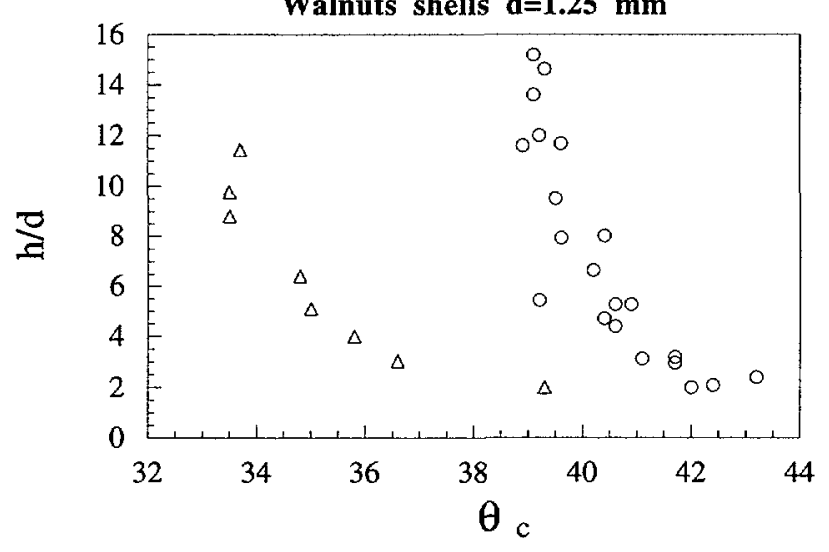

Fig. 9. - Variation of the effective static (circles) and dynamical (triangles) friction angle with the thickness of the pile $h / d$. a) experiments with glass beads of mean diameter $d=0.5 \mathrm{~mm}$. b) experiments with crashed walnuts shells of mean size $d=1.25 \mathrm{~mm}$.

and $\theta=25^{\circ}$ lies on the right side of the triangles. However, if one assumes that the curve given by the triangles represents the variation of the dynamic friction angle inside the material, one deduces from Figure $9 \mathrm{a}$ that a steady flow is not possible in the first 4 layers of material where the line $\theta=25^{\circ}$ lies on the left side of the triangles. Close to the bed a quasi-static region should then develop which will be surmounted by a layer of flowing material. Such quasi-static regions are indeed experimentally observed [14] and could thus be explained by the increase of the dynamic friction angle close to the rough bed.

\section{Conclusions}

The initiation of the flow of granular materials on a rough inclined plane has been studied both experimentally and by numerical simulations. The experiments consisted in slowly inclining a long rough bed on which a layer of granular material of constant depth was resting, until a big avalanche occurred. The critical angle at which the flow starts was found to depend on the initial depth of the pile, being greater for thin piles than for thick ones. These results can be 
interpreted as an increase of the internal friction coefficient of the medium close to the bed. We have performed two-dimensional experiments using cylinders as well as three-dimensional experiments with glass beads and crushed walnuts shells. For all the cases that were studied an increase of the shear strength was observed in the layers close to the bed, showing that this behavior is robust and independent of the shape or the nature of the particulate material.

Molecular-dynamics simulations have permitted us to carefully study the motion that takes place inside the pile when it starts to flow and also to obtain precise measurements of the dilatation of the medium. This study has revealed an increase of the coefficient of dilatancy close to the bed which is entirely correlated with the increase of the friction angle. The role of the bed roughness can thus be explained as follows. Close to the bed the particles are strongly affected by the rigidity of the bed such that they do not have much freedom to alter their arrangement. Thus in order to shear close to the bed the medium has to dilate even more, yielding a higher coefficient of friction.

We can conclude from this study that a rough bed strongly influences the dynamics of the flow of granular materials on an inclined plane, the bed being regular or not, and that this influence can be modelled by a variable internal friction coefficient, which increases close to the bottom surface.

\section{Acknowledgments}

The authors wish to thank Stuart B. Savage for introducing them to the world of granular media, and for many helpful comments and discussions. We have also benefited from fruitful and stimulating discussions with James W. Vallance and Ricardo Gutfraind. We thank Otis R. W. Walton for his help during the development of the molecular dynamics code. Special thanks go to Claudio Navas for his technical assistance and to Martin Serrer for the visualization software used during this work. We are grateful to Institut Français du Pétrole (IFP) and the National Science and Engineering Research Council of Canada (NSERC) for financial support.

\section{References}

[1] Jaeger H. M. and Nagel S. R., Science 255 (1992) 1523.

[2] Jaeger H. M., Liu C. and Nagel S. R., Phys. Rev. Lett. 62 (1989) 40.

[3] Rajchenbach J., Phys. Rev. Lett. 65 (1990) 2221.

[4] Morales-Gambo E., Lomnitz-Adler J., Romero-Rochin V., Chicharro-Serra R. and PeraltaFabi R., Phys. Rev. E 47 (1993) 2227.

[5] Caponeri M., Douady S., Fauve S. and Laroche C., Dynamics of avalanches in a rotating cylinder, In "Mobile particulate systems", NATO ASI Series E, E. Guazzelli and L. Oger Eds. (The Netherlands: Kluwer Academic Publishers, 1995).

[6] Reynolds O., Philos. Mag. 20 (1885) 469.

[7] Goddard J. D. and Bashir Y. M., On Reynolds Dilatancy, In "Recent developments in structured continua", vol. 2, D. De Kee and P. N. Kaloni Eds. (London, Longman Scientific and Technical, 1990).

[8] Rowe P. W., Proc. R. Soc. London Ser. A 269 (1962) 500.

[9] Bashir Y. M. and Goddard J. D., J. Rheol. 35 (1991) 849. 
[10] Goddard J. D., New theoretical estimates for dilatancy in granular materials, In "Theoretical and applied rheology", Proc. XI Int. Congr. of Rheology, P. Moldenaers and R. Keuning Eds. (Elsevier, Amsterdam, 1992).

[11] Balendran B. and Nemat-Nasser S., J. Mech. Phys. Solids 41 (1993) 573.

[12] Augenstein D.A. and Hogg R., Powder Tech. 19 (1978) 205.

[13] Savage S.B., Granular flows down rough inclines: review and extension, In "Mechanics of granular materials: new models and constitutive relations", J. T. Jenkins and M. Satake Eds. (Elsevier, Amsterdam, 1983).

[14] Drake T. J, J. Geophys. Res. 95 (1990) 8681.

[15] Vallance J. W., experimental and field studies related to the behavior of granular mass flows and the characteristics of their deposits, Ph.D Thesis (Michigan Technological University, 1994).

[16] Riguidel F. X., Jullien R., Ristow G., Hansen A. and Bideau D., J. Phys. I France 4 (1994) 261.

[17] Campbell C. S., Annu. Rev. Fluid Mech. 22 (1990) 57.

[18] Walton O. R., Numerical simulation of inelastic, frictional particle-particle interactions, in "Particulate two-phase flows", M. C. Rocco Ed. (Boston, Butterworth-Heinemann, 1993).

[19] Savage S. B., Investigations of some particulate flows by granular dynamics simulations, in "Recent advances in Mechanics of Structured Continua II", M. Massoudi and K. R. Rajogopal Eds. (American Society of Mechanical Engineers, 1993).

[20] Savage S. B. and Dai R., Mech. Mater. 16 (1993) 225.

[21] Dai R., Granular flows studies through kinetic theory and numerical simulation approaches, Ph.D Thesis (Department of Civil engineering and applied mechanics, Mcgill University, 1993).

[22] Huang Z. J., Computer modelling and simulations of mechanics of granular materials, Ph.D. Thesis (Department of Civil engineering and applied mechanics, Mcgill University, 1994).

[23] Walton O. R. and Braun R. L., J. Rheol. 30 (1986) 949.

[24] Bernal J. D., Proc. R. Soc. London Ser. A 280 (1964) 299.

[25] Mühlhaus H. B. and Vardoulakis I., Géotechnique 37 (1987) 271.

[26] Bardet J. P. and Proubet J., Géotechnique 41 (1991) 599.

[27] Jaeger H. M., Liu C. H., Nagel S. R. and Witten T. A., Europhys. Lett. 11 (1990) 619. 